\title{
Study on Extreme Risk Evaluation of Pork Price Fluctuation in Beijing City
}

\author{
Lei $\mathrm{Xu}^{1,2}$, Bingbing Wang ${ }^{1,2}$ \\ ${ }^{1}$ Agricultural Information Institute, Chinese Academy of Agricultural Sciences, Beijing 100081, \\ China \\ ${ }^{2}$ Center for Science and Technology Economic Policy, Chinese Academy of Agricultural Sciences, \\ Beijing 100081, China

\section{北京市猪肉价格波动极端风险度量研究 \\ 徐磊 ${ }^{1,2}$, 王兵兵 ${ }^{1,2}$ \\ ${ }^{1}$ 中国农业科学院农业信息研究所, 北京 100081 , 中国 \\ ${ }^{2}$ 中国农业科学院科技经济政策研究中心, 北京 100081 , 中国}

\begin{abstract}
In this paper, the tail distribution of pork market price fluctuation is fitted effectively based on the peaks over threshold (POT) model, and the Generalized Pareto Distribution (GPD) of extreme risk is obtained, and VaR (value at risk) method is introduced to measure the extreme risk of price fluctuation. Results show that using the POT model fromextreme value theory (EVT) to evaluate the extreme risk of pork market price is appropriate. When extreme risk in a one-hundred-year occurrence exposed to the market, the risk of surging pork price in Beijing city is higher than the downside risk, and the maximum monthly upside price reaches $35.8 \%$, corresponding to the drop of maximum amplitude to $32.05 \%$.
\end{abstract}

Keywords: Pork price, Extreme risk, Peaks over threshold model, VaR, Beijing city.

\section{摘要}

本文引入极值理论 POT 模型对北京市 猪肉市场价格波动尾部分布进行了有效拟 合, 获得了价格波动极端风险广义帕累托 分布 (GPD) 函数, 同时运用风险价值 (VaR) 方法计算极端风险值。研究表明:
运用极值理论 POT 模型度量猪肉市场价格 波动极端风险是适合的。当市场遭遇百年 一遇极端风险时, 北京市猪肉市场价格上 涨风险高于下跌风险, 月度间最大上涨幅 度达到 $35.8 \%$, 最大下跌幅度为 $32.05 \%$ 。

关键词: 猪肉价格; 极端风险; 超越阈值 模型; 风险价值; 北京市

\section{1 引言}

随着市场化改革的不断深入和国际化 进程的不断加快, 各种传统和非传统风险 不断叠加凸显, 我国部分农产品频现价格 暴涨暴跌现象, 尤其是猪肉价格 “过山车” 式大幅周期性波动 (“巨灾”), 对生猪产业 和居民消费均造成较大影响。据农业农村 部监测, 2000-2006 年期间, 我国猪肉集 贸市场平均价格月度间波动幅度多维持在 $5 \%$ 以内, 仅有 6 个月超过 5\%; 但 2007-2014 年期间, 月度间波动幅度位于 5\%-10\%区间 的明显增多, 有 34 个月超过 $5 \%$, 有 8 个月 份超过 10\%。特别是 2014 年年初以来遭遇 了史上最惨 “猪周期” , 据国家发展改革 委监测, 2014 年 1 月至 2015 年 5 月, 全国 猪粮比价连续 71 周低于 $6: 1$ 盈亏平衡点, 生猪养殖持续亏损。可以说, 如何有效管 控猪肉价格波动的极端风险 ( “巨灾”) 已 经成为社会关注的热点、管理决策的重点 和理论研究的难点。从世界各国地震、洪 
涝、台风等自然灾害巨灾风险管理实践的 经验和教训来看, 能否建立适合的巨灾风 险度量模型在很大程度上决定着风险管理 的最终成败。以此类推, 针对猪肉价格波 动的极端风险 ( “巨灾”), 建立精准科学的 极端风险度量模型是对其实施有效管控的 前提和基础。

目前国内外学者对猪肉价格波动极端 风险度量的研究较少, 大量文献主要集中 如何确定猪肉价格的波动周期 ${ }^{[1-8]}$ 以及分析 猪肉价格波动的影响因素 ${ }^{[9-15]}$ 。不可否认, 这些成果为本研究提供了很好的理论和方 法借鉴, 但国内外既有的研究并没有从猪 肉价格波动风险的内涵出发, 自然无法实 现猪肉价格波动极端风险的度量。此外, 起源于 20 世纪 90 年代, 被众多金融机构 广泛采用度量金融市场风险, 也包括度量 操作风险的 VaR 方法 ${ }^{[16]}$, 被尝试引入农产 品市场价格波动风险研究中, 得出了一些 有意义的结论 ${ }^{[17-18]}$, 但既有的研究同时指出, 传统的 VaR 并不适合极端风险的情形, 其 结果会出现偏差 ${ }^{[19]}$ 。具体而言, 基于均值 理论的普适模型在常规风险的度量中得到 较好的应用 ${ }^{[20]}$, 但对于具有 “低频高损” 特征、处于尾部分布的 “巨灾” (包括农产 品市场价格的异动) 风险却存在着明显的 局限, 往往无能为力。

因此, 本文基于传统的 VaR 方法, 引 入现代极值理论 (EVT) 中的超越阈值 ( $\mathrm{POT}$ ) 方法以期实现对价格波动尾部分布 的有效拟合, 从而获得价格波动极端风险 广义帕累托分布 (GPD) 模型, 同时以北京 市猪肉市场价格为例, 根据拟合的分布函 数模拟计算发生百年一遇情形时北京市猪 肉市场价格上涨和下跌的最大幅度。

\section{2 方法模型}

\section{1 构建猪肉价格波动时间序列}

本文采用月度居民消费价格指数（CPI） 对猪肉市场价格进行修正, 剔除货币因素 对价格波动的影响。数据转换公式为:

$$
\mathrm{p}_{\mathrm{t}}^{\prime}=\frac{\mathrm{p}_{\mathrm{t}}}{\mathrm{cpi}_{\mathrm{t}}}
$$

上式中， $\mathrm{p}_{\mathrm{t}}$ 表示猪肉市场第 $\mathrm{t}$ 期的价格， $\mathrm{cpi}_{\mathrm{t}}$ 表示地区第 $\mathrm{t}$ 期的居民消费价格指数, $\mathrm{p}_{\mathrm{t}}^{\prime}$ 表示剔除货币、通胀等因素后的第 $\mathrm{t}$ 期实 际价格。

基于此, 本研究将市场价格时间序列 构建成一个月度间环比增长率序列。公式 如下:

$$
\mathrm{R}_{\mathrm{i}}=\frac{\mathrm{p}_{\mathrm{t}}^{\prime}-\mathrm{p}_{\mathrm{t}-1}^{\prime}}{\mathrm{p}_{\mathrm{t}-1}^{\prime}}
$$

上式中, $\mathrm{R}_{\mathrm{i}}$ 为猪肉市场价格的环比增长 率, 作为猪肉价格波动的衡量指标。

\section{2 拟合猪肉价格波动时间序列尾部分布}

极值理论是次序统计学的一门分支, 主 要研究的是极值分布及其特征, 尤其是分 布的尾部特征 ${ }^{[21]}$ 。根据现代极值统计理论 ( EVT) 中的 POT 模型 ( peaks over threshold, 超越阀值模型), 基于猪肉市 场价格的环比增长率 $\mathrm{R}_{\mathrm{i}}$ 时间序列, 通过运用 平均超限函数图以确定阈值 $\mathrm{u}$, 进而对超越 阈值之上的样本 (即为猪肉市场价格波动 的尾部分布) 进行拟合, 其分布将服从广 义帕累托分布 ( generalized pareto distribution, GPD), 具体分布函数形式 为:

$$
\begin{gathered}
\mathrm{G}_{\xi, \sigma}(\mathrm{y})=\left\{\begin{array}{l}
1-\left(1+\frac{\xi y}{\sigma}\right)^{-1 / \xi}, \xi \neq 0 \\
1-\exp \left(-\frac{y}{\sigma}\right), \xi=1
\end{array}\right. \\
(\xi \geq 0 \text { 时, } \sigma>0, \mathrm{y} \geq 0 ; \xi<0 \text { 时, }
\end{gathered}
$$

$0 \leq \mathrm{y} \leq-\frac{\sigma}{\xi}$ ), 其中 $\xi$ 为形状参数, $\sigma>0$ 为 尺度参数, 决定了分布尾部消失的速度, 是分布中极为重要的参数, $\mathrm{y}=\mathrm{x}-\mathrm{u}$ 。从 而获得了猪肉市场价格上涨极端风险的分 布函数。

\section{3 运用 VaR 计算猪肉价格波动极端风 险值}

风险价值 (Value at Risk, VaR) 从统 计的意义上讲, 是指面临正常的市场波动 时处于风险状态的价值, 即在给定的置信 水平和一定的持有期限内, 预期的最大损 失量。其数学定义是: 设 $\mathrm{X}$ 是描述资产组 合损失的随机变量, $F(X)$ 是其概率分布函 数, 置信水平为 $\alpha$, 则 
$\operatorname{VaR}(\alpha)=-\min \{\mathrm{x} \mid \mathrm{F}(\mathrm{x}) \geq \mathrm{A}\}$

基于猪肉市场价格上涨极端风险的分布 函数为 $\mathrm{F}(\mathrm{x}), \mathrm{x}$ 为价格波动值, $\mathrm{VaR}$ 为在正 常市场条件下和一定置信水平下, 猪肉市 场价格可能偏离其预期价格的最大程度 (即最大的价格风险)。

给定一个置信水平 $\mathrm{p}$, 通过平均超限函 数图确定的阈值 $\mathrm{u}$, 对公式 $\mathrm{F}(\mathrm{x})$ 求其的逆函 数, 得到该置信水平下的上涨极端风险的 $\mathrm{VaR}_{\mathrm{p}}$, 具体函数表达式:

$\operatorname{VaR}_{\mathrm{p}}=\left\{\begin{array}{l}u+\frac{\sigma}{\xi}\left\{\left[\frac{\mathrm{n}}{\mathrm{N}_{\mathrm{u}}}(1-\mathrm{p})\right]^{-\xi}-1\right\}, \xi \neq 0 \\ \mathrm{u}-\sigma \ln \left[\frac{\mathrm{n}}{\mathrm{N}_{\mathrm{u}}}(1-\mathrm{p})\right], \quad \xi=0\end{array}\right.$

以此类推, 将环比增长率 $\mathrm{R}_{\mathrm{i}}$ 中的负值转 正, 然后从大到小排列, 通过平均超限函 数图确定的阈值 $u^{\prime}$ 进行分布拟和, 获得猪肉 市场价格下跌极端风险的分布函数, 计算 得到置信水平 $\mathrm{p}$ 的下跌极端风险的 $\mathrm{VaR}_{\mathrm{p}}^{\prime}$ 。

\section{3 实证分析}

\section{1 数据的简单统计描述}

本文以北京市猪肉市场价格的月度数据 为研究对象, 选取 2000 年 1 月至 2017 年 7 月之间的数据作为样本数据进行建模。原 始数据来源于中国畜牧业信息网以及中国 国家统计局。本研究中的猪肉价格特指去 皮带骨猪肉价格。采用月度居民消费价格 指数 (CPI) 对北京地区的猪肉市场价格进
行修正, 剔除货币因素对价格波动的影响, 得到实际的市场价格, 如图 1 所示。

总的来看, 北京市猪肉价格呈现出震荡 上行的态势, 从 2000 年 1 月到 2017 年 7 月, 北京市猪肉价格最高为 29.88 元/千克, 最低为 8.06 元/千克, 价格差高达 21.82 元, 变动的相对幅度较大 (图 1)。

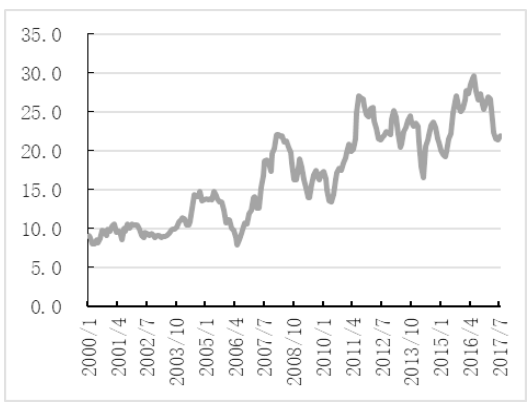

图 1. 北京猪肉市场实际价格走势图

为反映北京市猪肉价格波动风险的总体 特征, 本文进一步对实际价格时间序列作 简单统计量分析, 其结果如表 1 所示。由 于样本峰度大于 3 , 呈现出的 “尖峰厚尾” 特征, 而且 $\mathrm{J}-\mathrm{B}$ 统计量的数据都足够大, 这说明北京市猪肉市场价格环比增长率序 列不服从正态分布的情况, 因此, 适合应 用极值理论对猪肉市场价格波动极端风险 进行度量。

表 1. 北京地区猪肉市场价格波动率的描述性统计

\begin{tabular}{|c|c|c|c|c|c|c|c|c|}
\hline 均值 & 中位数 & 最大值 & 最小值 & 标准差 & 偏度 & 峰度 & JB 量 & $\mathrm{P}$ \\
\hline 0.0062 & 0.0058 & 0.2427 & -0.1520 & 0.0638 & 0.5446 & 3. 8960 & 17. 4033 & 0 \\
\hline
\end{tabular}

\subsection{POT 模型阈值的确定和 GPD 分布的 参数估计}

本文运用 POT 模型对北京市猪肉市场价 格波动率样本分布的尾部进行拟合, 并采 用极大似然估计法 (MLE) 对模型参数进行 估计。其中阈值 $u$ 的确定极为关键, 过高 的阈值会使可利用的样本容量过少, 从而 导致估计参数的方差偏高; 而阈值过低又 会使模型的渐近性得不到满足, 产生有偏 估计量。目前学术界通常采用平均超限函 数来确定阈值, 已有的研究结果表明, 如 果在某个数值之后的平均残差寿命图趋向
于线性时, 则该数值即为样本的阈值。因 此, 本研究将通过平均超限函数图来确定 阈值, 然后通过 MLE 来估计 GPD 的参数, 从而获得北京市猪肉市场波动极端风险的 概率分布函数。

根据上述原则, 确定北京地区猪肉市场 价格上涨风险阈值, 同时将波动率中的负 波动率转正, 然后从大到小排列, 再次确 定下跌风险阈值。得到猪肉市场价格波动 上涨和下跌平均超限函数 (图 2 和图 3)。 
上涨

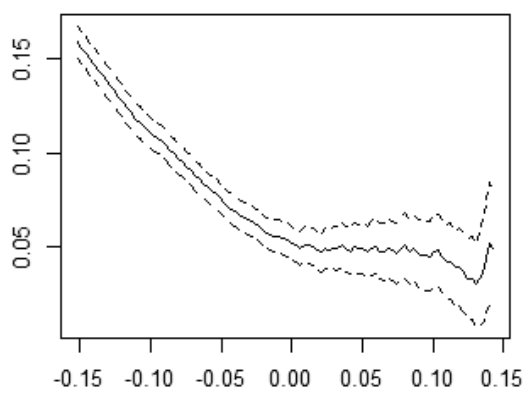

下跌

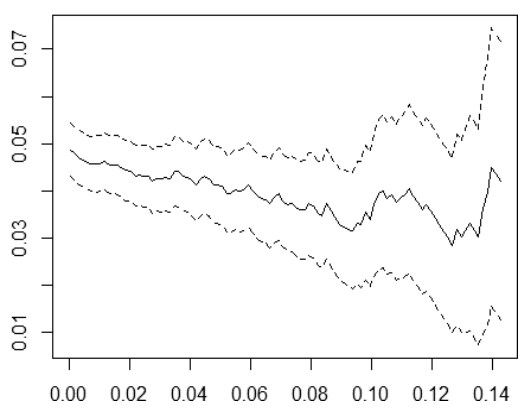

图 2. 北京猪肉市场价格波动率平均超限函数图
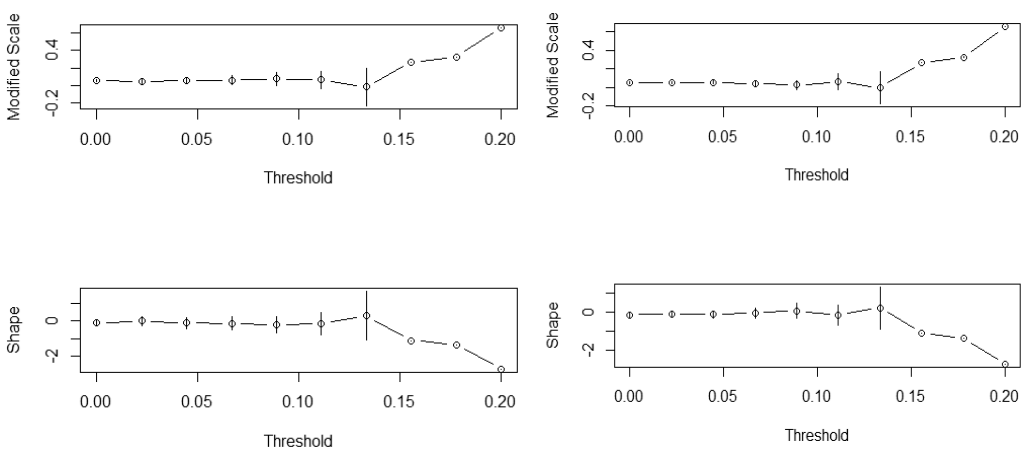

图 3. 北京猪肉市场价格波动率阈值选取范围

结合图 2 和图 3 确定阈值后, 运用 MLE 法对超越阈值之上样本的分布模型参数进 行估计, 得到北京市猪肉市场价格上涨和 下跌极端风险的广义帕累托分布函数参数 估计值（表 2)。

表 2. 北京猪肉市场价格波动率风险参数估计值

\begin{tabular}{cccc}
\hline & $\mathrm{u}$ & $\boldsymbol{\Sigma}$ & $\xi$ \\
\hline 上涨 & 0.13 & 0.01897 & 0.44736 \\
下跌 & 0.13 & 0.02138 & 0.31759 \\
\hline
\end{tabular}

为进一步检验上述模型分布的准确性, 需要对猪肉市场价格波动风险模型拟合的 效果进行诊断。本文结合拟合的广义帕累 托分布 (GPD) 和经验的概率图 (P-P 图)、 分位数图（Q-Q 图)、重现水平图（Return Level P1ot) 和概率密度函数图 (Density P1ot) 分别进行诊断比较, 结果见图 4。不 难发现, 概率图上的点基本处于对角线上; 分位数图中右上方的点离对角线有少许偏 离, 但从重现水平图来看仍然处于置信水
平之内, 说明数据与模型的偏离不大; 由 概率密度图可以看出, 分布函数的估计和 频率图拟合的也相对较好。因此总的来说, 样本拟合效果可以接受。

\section{3 北京市猪肉价格波动极端风险 VaR}

由于使用的是月度样本数据, 所以当市 场发生百年一遇时 “巨灾” 时, $\mathrm{p}$ 值应该取 $1-1 / 1200=99.92 \%$, 同时将参数估计结果代 入相应公式, 可求得北京猪肉市场价格波 动极端风险值 VaR (表 3 )。

表 3. 北京猪肉市场价格波动风险值 单位: \%

\begin{tabular}{ccc}
\hline $\mathrm{VaR}$ (百年一遇) & 上涨 & 下跌 \\
\hline 北京 & 35.80 & 32.05 \\
\hline
\end{tabular}

从表 3 中不难发现: 当市场遭遇百年一 遇极端风险时, 北京市猪肉市场价格上涨 的幅度要高于下跌幅度, 即月度间最大上 涨幅度达到 $35.8 \%$, 最大下跌幅度为 $32.05 \%$ 。 

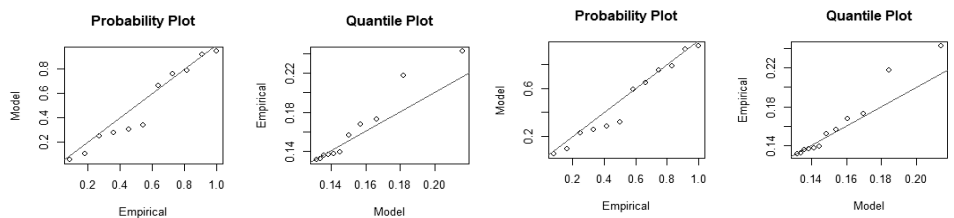

Model
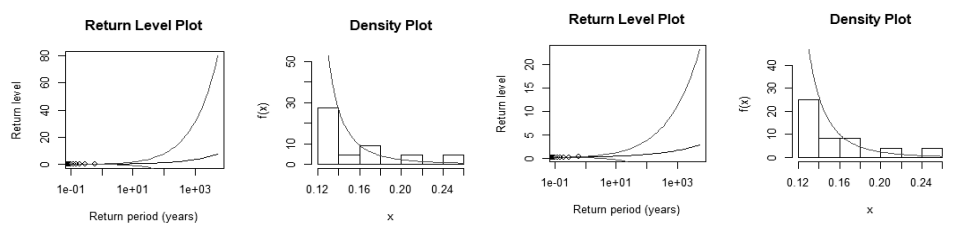

图 4. 北京猪肉市场价格波动风险分布拟合诊断图

\section{4 简要结论和政策建议}

本文在传统 VaR 方法的基础上，构建了 基于 POT 模型的猪肉价格波动极端风险度 量模型, 当市场遭遇百年一遇的极端风险 时, 无论是上涨还是下跌, 北京市猪肉市 场价格的波动幅度均超过了 $30 \%$, 或将对生 猪产业和居民消费均造成较大影响。

为避免猪肉市场价格频繁出现异动, 加 强猪肉市场价格波动极端风险管理, 拟提 出以下政策建议: 一是加强和完善生猪产 业监测和预警。政府部门应完善生猪全产 业链统计监测工作, 对影响价格波动的各 类因素尽可能的纳入监测范围, 同时建立 健全政府公共信息服务平台, 及时发布和 传递市场信息, 有效引导市场自发调节供 需平衡。二是加快生猪养殖适度规模化发 展进程, 同时提高生产和经营主体风险管 理意识, 增强风险管控能力。三是创新猪 肉市场风险管理工具, 基于猪肉价格波动 极端风险度量技术, 创新和开发生猪期货 产品、猪肉价格保险、“保险+期货” 等市 场风险工具, 提高猪肉价格波动极端风险 管理的效率和效能。

\section{Acknowledgement}

This work was jointly funded by Beijing Municipal Natural Science Foundation (Grant No. 9172022) and Basal Research Fund for Central Scientific Research Institute (Grant No. JBYW-AII-2016-02).

\section{致谢}

本研究得到了北京市自然科学基金面上项 目（资助编号：9172022）和公益性科研院 所基本科研业务费项目（资助编号：JBYWAII-2016-02）的资助。

\section{参考文献}

[1] Harlow A A. The Hog Cycle and the Cobweb Theorem. American Journal of Agricultural Economics, 1960, 42(2): 842-853.

[2] Talpaz H. Multi-Frequency Cobweb Model: Decomposition of the Hog Cycle. American Journal of Agricultural Economics, 1974, 56(1):38-49.

[3] Futrell G A, Grimes G. Understanding hog production and price cycles. Agriculture and Home Economics, 1978, (11):1-5.

[4] 李秉龙,何秋红.中国猪肉价格短期波动 及其原因分析.农业经济问题, 2007(10): 18-22.

[5] 董玲. 我国猪肉价格波动研究.内蒙古农 业大学, 2010.

[6] 于少东.北京市猪肉价格波动周期分析. 农业经济问题,2012(02):75-78.

[7] Parker P S, Shonkwiler J S. On the centenary of the German hog cycle: new findings. European Review of Agricultural Economics, 2014, 41(1): 4761.

[8] 郭刚奇.基于 $\mathrm{ARCH}$ 模型的猪肉价格波 动短期特征分析. 经济问题,2017(11):95100. 
[9] 辛贤,谭向勇.中国生猪和猪肉价格波动 因素测定.中国农村经济,1999(5):28-34.

[10] Parcell J L. An Empirical Analysis of the Demand for Wholesale Pork Primals: Seasonality and Structural Change. Journal of Agricultural \& Resource Economics, 2003, 28 (2):335-348.

[11] 吕杰,綦颖. 生猪市场价格周期性波动的 经济学分析. 农业经济问题,2017(7):8992.

[12] 柯柄生,韩一军.对近期猪肉价格上涨情 况的分析判断及建议. 农业展望, 2007(7):10-11.

[13] 邓攀.安徽省生猪市场价格波动成因分 析及对策. 安徽农业大学, 2010 .

[14] 吴登生.生猪价格波动特征及影响事件 的混合分析模型与实证.系统工程理论 与实践,2011(11): 2033-2042.

[15] 宋长鸣.非线性非均衡蛛网模型框架下 猪肉价格循环波动研究——基于可变 参数模型的实证. 华中农业大学学报(社 会科学版),2016(06):1-7.

[16] He M L, Qing S T, Mo J M, Gao X. Connection Parameters of Heavy-tailed Operational Risk Measurement Model and Management Model. Journal of Risk Analysis and Crisis Response, 2016, 6(3):122-134.

[17] 张峭,王川,王克. 我国畜产品市场价格风 险度量与分析.经济问题,2010,(3):90-94.

[18] 熊巍,祁春节. 基于 $\mathrm{VaR}$ 的果蔬农产品价 格的风险度量. 统计与决策, 2013 , (21):126-130.

[19] Morgan J P. Risk Metrics-Technical Document (4th edition).New York: JP Morgan, 1996.

[20] HaoJ. Modeling and financing weather risk: three essays.Lexington; University of Kentucky, 2005.

[21] 实用极值统计方法. 天津科学技术出版 社,史道济著,2006. 